dade do espaço e frequente falta de verbas necessárias à efectivação de alguns trabalhos assim como à aquisição e manutenção de material.

As instalações encontram-se dispersas pelas várias dependências da FCL onde funcionavam os núcleos ini- ciais, bem como no $1 .^{\circ}$ andar do Instituto de Investigação Científica Bento da Rocha Cabral, especialmente cedido em 1976 em virtude da referida exiguidade de espaço disponível na Faculdade, e no qual funcionam parcialmente as linhas 3 e 4 .

\section{QUIMIOMETRIA: Ciência das Medições em Química}

Com o avanço tecnológico em domínios como, a electrónica, a física, ou a ciência dos computadores, a grande linha de desenvolvimento da Química Analítica centrou-se na expansão dos chamados "Métodos Instrumentais". No entanto, uma vez que a todas as medições analíticas está associado um certo grau de incerteza e que os resultados analíticos são geralmente derivados de fórmulas matemáticas, é difícil conceber a Química Analítica dissociada do Cálculo Matemático e da Estatística [1]. A aplicação à Química Analítica de métodos matemáticos e estatísticos não totalmente novos, tem vindo a ser incrementada pelo aparecimento dos computadores, instrumentos indispensáveis à Química Analítica moderna, para aquisição, armazenamento, fornecimento e processamento de dados.

Assim, desde os anos 70 tem vindo a desenvolver-se um novo ramo da Química, a Quimiometria, internacionalmente conhecida por "Chemometrics", o qual movimentando-se na interface entre a Química e a Matemática $\left[{ }^{2,3}\right]$, fornece a via racional que transforma os dados experimentais em informação.

A Chemometrics Society, fundada em 1974 por S. Wold-Section for Chemometrics, Institute of Organic Chemistry, Universety of Umea - Suécia e B.R. Kowalski - Laboratory of Chemometrics, Universidade de Washington Seattle, USA, define Quimiometria: “O ramo da Química que usa métodos matemáticos e estatísticos para:

a) Delinear e seleccionar as experiências e os modos de proceder óptimos;

b) Extrair o máximo, em qualidade e quantidade de informação a partir da análise de conjuntos complexos de dados.

No campo da Química Analítica, a Quimiometria é usada para obter a informação relevante, óptima, dos sistemas materiais $\left[{ }^{4}\right]$.

A combinação adequada da Quimiometria e da Informática favorece a transição da Química Analítica, de simples fornecedora de dados, para a Química Analítica que resolve efectivamente os problemas.

A conceptualização de um problema analítico envolve a selecção e utilização de um método válido, num programa válido, sobre uma amostra válida. É missão do Químico Analista tomar as decisões adequadas, óptimas, em cada passo do problema analítico. Para tal, é necessário por um lado, um conhecimento profundo do problema químico: "que objectivo se pretende atingir ao reali-
M.F. CAMÕES (a), M.H. FLORÊNCIO (b), A.M. NUNES DOS SANTOS (b)

zar as experiências e ao recolher os dados experimentais?"'. Por outro lado, é necessário um conhecimento de quais os métodos matemáticos-estatísticos-numéricos que mais de perto servem à resolução do problema.

Para um Químico Analista ser bem sucedido, desde a formulação de um problema até à sua resolução, deve dominar os princípios e a filosofia tanto do problema químico e da técnica experimental como da metodologia a seguir para a análise de dados.

Os problemas com que a Química Analítica se debate podem frequentemente ser reformulados em termos de problemas de Quimiometria [5,6].

Descriminam-se a seguir alguns exemplos:

1 - Problemas Univariantes: Estudo de uma propriedade função de uma variável.

Um tratamento univariante ignora, quer outras variáveis que podem influenciar o resultado de um modo significativo, quer possíveis interacçð̃es de variáveis.

Um tratamento univariante tem uma representação gráfica a duas dimensões que pode ser enganosa, como a seguir se evidencia esquematicamente [ $\left.{ }^{6}\right]$.
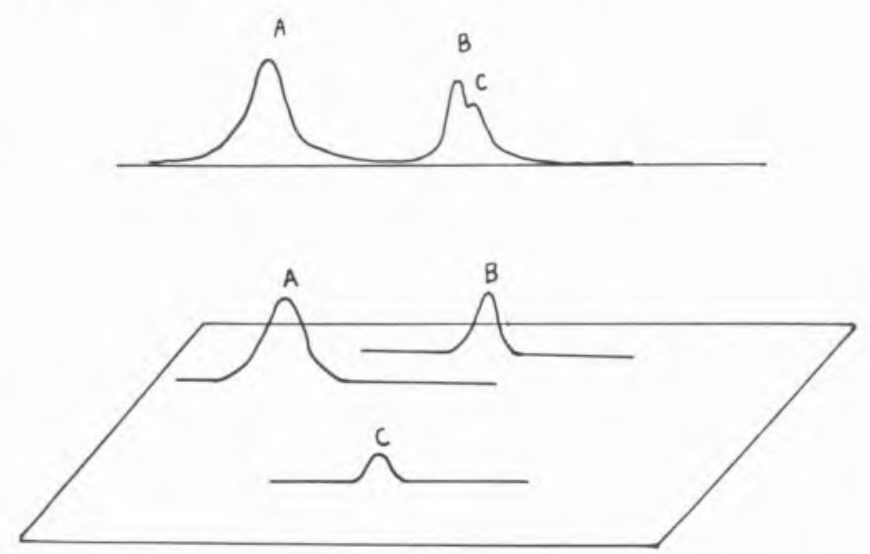

No espaço bidimensional, o pico $\mathrm{C}$ está mais próximo do pico B que do pico A ( $\left.{ }^{\mathrm{a}}\right)$.

No espaço tridimensional pode observar-se que na realidade o pico $\mathrm{C}$ se encontra mais próximo do $\mathrm{A}$ (b).

* Os autores são membros da Chemometrics Society sendo um deles (M.H.F.) o contacto nacional.

(a) Departamento de Quimica, F.C.U.L., 1294 LISBOA CODEX

(b) Dept. de Engenharia Quimica, F.C.T., Universidade Nova de Lisboa, 2825 MONTE DA CAPARICA 
2 - Problemas de ajustamento de curvas ("curve fitting'"):

Escolha de um modelo matemático com parâmetros ajustáveis, que descreve os dados experimentais [ $\left.{ }^{7}\right]$.

Como exemplo podem desenvolver-se equações para cálculo de largura, altura, ou posição de picos de modo a minimizar os desvios entre os dados calculados e os dados experimentais.

3 - Problemas de descriminação de modelos:

Inclui os problemas anteriores e ainda o de delinear as experiências cujos resultados melhor se insiram em determinados modelos [ $\left.{ }^{8}\right]$.

4 - Problemas de classificação:

Determinar se um dado objecto é mais semelhante a um grupo de objectos que a outro. Uma medida quantitativa de semelhança pode ser expressa em termos de distância ou correlação entre propriedades características [ $\left.{ }^{9}\right]$.

Será esta semelhança suficientemente significativa para concluir que o objecto pertence de facto ao grupo? Quais as variáveis relevantes para a classificação e que, portanto, devem ser objecto de medição?

5 - Problemas de agrupamento: ("clustering"'):

Procura agrupar objectos de um modo natural, com base na medição de propriedades características [ $\left.{ }^{5,9}\right]$.

6 - Problemas de optimização:

Quais os melhores parâmetros?

Quais as melhores condiçð̃es experimentais?

Qual o melhor, óptimo, valor de um parâmetro?

Estas questões podem, por vezes, surgir simultaneamente [ $\left.{ }^{9}\right]$.

A solução destes problemas passa pelo uso de técnicas matemáticas e estatísticas tais como [5]:

(a) Modelos estatísticos univariantes - distribuição de Poisson, $\mathrm{F}, \mathrm{T}, \mathrm{X}^{2}, \ldots$

(b) Correlação de regressão linear e não linear;

(c) Análise espectral, "time-series analysis";

(d) Métodos multivariantes:

- Regressão múltipla; análise factorial dos componentes principais;

- Reconhecimento de padrões (“'Pattern Recognition'”), análise descriminante, análise de grupos ("clustering"');

- Métodos de minimização e maximização.

(e) Análise operacional, teoria dos sistemas - métodos gráficos.
A Quimiometria é já considerada como um domínio diferenciado da Química, merecendo inclusivamente uma secção autónoma do jornal científico Analytical Chemistry.

Após fases iniciais de:

1 - Sistematização de métodos matemáticos de análise de dados;

2 - Utilização, investigação e desenvolvimento desses métodos para a resolução de problemas químicos; está-se actualmente numa fase de introdução da Quimiometria no ensino, quer a nível graduado quer pós-graduado.

São já várias as Universidades, na Europa e nos Estados Unidos da América, em que a Quimiometria faz parte do curriculum regular dos cursos de Química e de Química Analítica [10-13].

O Grupo de Trabalho de Química Analítica da Federação Europeia das Sociedades de Química (WPAC/ /FECS) levou recentemente a efeito um inquérito sobre “A Educação em Química Analítica”, cujos resultados revelam já a implantação de Quimiometria, inclusivamente ao nível de diplomas de pós-graduação.

Em face do exposto, para uma formação equilibrada do Químico Analista, os autores reconhecem a premência da inclusão, com lugar de destaque, da Quimiometria nos curricula dos cursos de Química e de Engenharia Química, bem como em seminários e cursos de reciclagem.

\section{REFERENNCIAS}

[1] B. R. Kowalski, Anal. Chem. 52, 112 R (1980).

[2] B. R. Kowalski, Chemistry and Industry, 22, 882 (1978).

[3] I. E. Frank e B. R. Kowalski, Anal. Chem., 54, 232 R (1982).

[4] B R. Kowalski, Trends in Analytical Chemistry, 1, 71 (1978).

[5] S. Wold, Chemometrics Newsletter n. ${ }^{\circ} 3,4,1977$.

[6] B. R. Kowalski, Chemometrics Newsletter n. ${ }^{\circ}$ 3, 10, 1977.

[7] G. Kateman e F. W. Pijpers, Quality Control in Analytical Chemistry, J. Wiley, New York, 1981.

[8] H. Hofmann, Chemical Reaction Engineering, 519 (1970).

[9] D. L. Massart, A. Dijkstra e L. Kaufmann, Evaluation and Optimization of Laboratory Methods and Analytical Procedures, Elsevier, Amsterdam 1978.

[10] B. G. M. Vandeginste, Analytica Chimica Acta, 150, 199 (1983).

[11] D. G. Howery e R. F. Hirsh, J. Chem. Ed., 60, 656 (1983).

[12] D. L. Massart, Fresenius Z. Anal. Chem., 305, 113 (1981).

[13] H. A. van't Klooster, Chemisch Magazine, 509 (1982).

\section{COLABORE COM A SOCIEDADE}

\section{NÃO ATRASE O PAGAMENTO DAS SUAS QUOTAS}

